\title{
Single-Mode Modified Tapered Fiber Structure Functionalized With GO-PVA Composite Layer for Relative Humidity Sensing
}

\author{
Aneez SYUHADA ${ }^{1,4}$, Muhammad Salleh SHAMSUDIN ${ }^{1,2,4}$, Suzairi DAUD ${ }^{1}$, \\ Ganesan KRISHNAN ${ }^{1,4}$, Sulaiman Wadi HARUN ${ }^{3}$, and Muhammad Safwan Abd. AZIZ ${ }^{1,4^{*}}$ \\ ${ }^{1}$ Department of Physics, Faculty of Science, Universiti Teknologi Malaysia, Johor Bahru 81310, Malaysia \\ ${ }^{2}$ School of Engineering, Faculty of Engineering and Physical Sciences, University of Southampton Malaysia, Iskandar \\ Puteri 79200, Malaysia \\ ${ }^{3}$ Department of Electrical Engineering, University of Malaya, Kuala Lumpur 50603, Malaysia \\ ${ }^{4}$ Laser Center, Ibnu-Sina Institute for Scientific and Industrial Research (ISI-SIR), Universiti Teknologi Malaysia, Johor \\ Bahru 81310, Malaysia \\ "Corresponding author: Muhammad Safwan Abd. AZIZ_ E-mail: safwanaziz@utm.my
}

\begin{abstract}
A sensitive tapered optical fiber sensor incorporating graphene oxide (GO) and polyvinyl alcohol (PVA) composite film for the rapid measurement of changes in relative humidity was proposed and experimentally demonstrated. The sensing principle was based on the intensity modulation of the transmitted light induced by the refractive index changes of the sensitive coatings. The sensing region was obtained by tapering a section of single-mode optical fiber (SMF) from its original $125 \mu \mathrm{m}$ diameter down to $9.03 \mu \mathrm{m}$. The tapered structure was then modified through deposition of GO/PVA nanocomposites by using the dip-coating technique. The field emission scanning electron microscope (FESEM) and Raman spectroscopy were used to characterize the structure of the composite film. As evidenced by a Fourier transform infrared spectroscopy (FTIR) analysis, the presence of oxygen functional groups (such as $-\mathrm{OH}$ and $\mathrm{COOH}$ ) on the $\mathrm{GO}$ structure enabled the attachment of PVA molecules through hydrogen bonding and strong adhesion between $\mathrm{GO} / \mathrm{PVA}$ layers. The performance of the sensor was tested over a wide range ( $20 \% \mathrm{RH}$ to $99.9 \% \mathrm{RH})$ of relative humidity. The sensor showed a good response with its signal increasing linearly with the surrounding humidity. The tapered optical fiber sensor with the coating of GO/0.3 $\mathrm{g}$ PVA achieved the highest sensitivity [0.5290 RH (\%)]. The stability, repeatability, reversibility, as well as response time of the designated sensor were also measured and analyzed.
\end{abstract}

Keywords: Humidity sensor; tapered optical fiber; Graphene Oxide; PVA

Citation: Aneez SYUHADA, Muhammad Salleh SHAMSUDIN, Suzairi DAUD, Ganesan KRISHNAN, Sulaiman Wadi HARUN, and Muhammad Safwan Abd. AZIZ, "Single-Mode Modified Tapered Fiber Structure Functionalized With GO-PVA Composite Layer for Relative Humidity Sensing," Photonic Sensors, 2021, 11(3): 314-324.

\section{Introduction}

Knowledge in relative humidity $(\mathrm{RH})$, the precise amount of water vapor in the air expressed as a percentage of the quantity needed for saturation at the same temperature, helps scientists in designing applications to satisfy human needs.

Received: 30 October 2019 / Revised: 2 April 2020

(C) The Author(s) 2020. This article is published with open access at Springerlink.com

DOI: $10.1007 / \mathrm{s} 13320-020-0595-0$

Article type: Regular 
Measurements of $\mathrm{RH}$ are often expressed in percentage. The relation is determined by (1):

$$
R H(\%)=\frac{P_{v}}{P_{s}} \times 100
$$

where $P_{v}$ is described as the actual partial pressure of the moisture content in the air while $P_{s}$ is the saturated pressure of the moisture content in the air at constant temperature (Bar or $\mathrm{kPa}$ ) [1]. $\mathrm{RH}$ measuring sensors are used in agriculture, food processing, semiconductor processing, mining, textile, and pharmaceuticals industries. It is expected that the humidity sensor market will reach approximately USD 1.88 billion per annum by 2023 with a compound annual growth rate of $15 \%$ starting from 2017.

In the last decade, fiber-based sensors have attracted significant interest. Fiber-based sensing offers advantages over electrical and electronic sensing due to the predictable and controllable nature of the photonic behavior of the light. Fiber-based sensors can operate in hazardous settings and even those with a high risk of explosion [2]. Most importantly, it is unaffected by electromagnetic interference produced by electronic devices situated nearby the sensor [3]. Another advantage is the size of the fiber being relatively small, which is compact and space-saving. With easy and safe installation and operation, optical fiber sensors have become a popular research focus where their potential is being explored in many kinds of measuring purposes [4, 5]. Among the different types of fiber-based sensors, tapered optical fiber sensors are considered as one of the most facile and easy sensors to be fabricated. This type of sensor is fabricated by reducing the diameter of the fiber to allow the existence of evanescence waves that are highly sensitive with any changes happening in the surrounding medium. The ultrasensitive structure can be manipulated for different sensing applications.

The coating of fiber-based sensors with nano- structured elements is helpful as it provides high-impact improvements in the accuracy, reliability, stability, and practicality. The RH sensing mechanism in the tapered optical fiber sensor application involves the humidity sensitive coatings (hydrophilic materials) coated on the tapered region to enhance the transmission signal through the improvements on evanescent waves [6]. Some of the materials are used in the coating include polyvinyl alcohol (PVA) [7, 8], chitosan [9], metallic oxide film [10], and graphene oxide (GO) [11, 12]. With 880 gas sensor papers recorded in September, 2018, GO represents an important potential material for discussing [13]. This 2-dimension (2D) material is reliable due to its morphological property, which has a high surface-to-volume ratio due to its layered honeycomb structure and excellent conductivity, and the surface can easily be modified by its functional groups. GO sheets contain abundant reactive oxygen functional groups, epoxy groups $(\mathrm{C}-\mathrm{O}-\mathrm{C})$, carboxyl groups $(-\mathrm{COOH})$, carbonyl groups $(\mathrm{C}=\mathrm{O})$, and hydroxyl groups $(-\mathrm{OH})$ at the edge of the sheets, making it strongly hydrophilic [14]. The involvement of hydrophilic property supports the material to swell physically and the refractive index changes corresponding to the shift in $\mathrm{RH}$. Incorporation of the GO with the inorganic semiconductor or polymer such as the PVA is a suitable way to further enhance the former's film-forming characteristics. The polymer coatings have the advantages of excellent performance with long-term stability [15], making it a good choice to be exploited together with the GO to form a stable nanocomposite film. The presence of oxygen functional groups in the graphene oxide structure makes it possible for intercalation of the PVA to happen between the layers of the GO to form a composite film as illustrated in Fig. 1. Reports have been made regarding the largely improved mechanical properties of the PVA when the GO is added into the matrix [16-18]. 


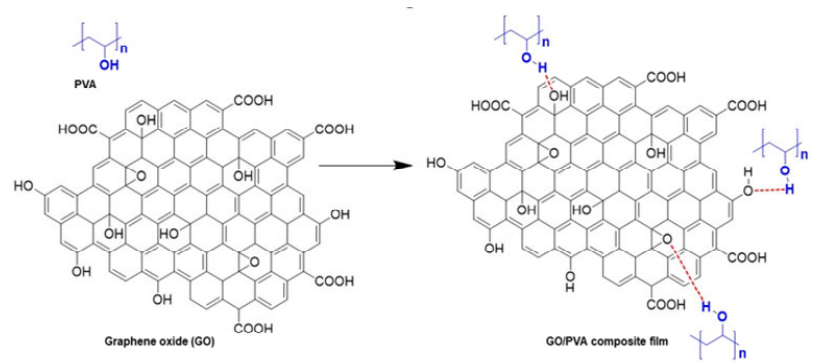

Fig. 1 Molecular structure of the GO before and after the intercalation with the PVA.

In this study, we reported on the absorbance response of the tapered optical fiber coated with GO/PVA nanocomposites layer exposed with the humidity level ranging from $20 \% \mathrm{RH}$ to $99.9 \% \mathrm{RH}$. The stability, repeatability, reversibility, and response time of the developed sensor were tested and analyzed.

\section{Mechanism of the tapered optical fiber sensor}

By using the knowledge of the total internal reflection principle and evanescence waves' interaction, tapered fiber sensors are made by reducing the diameter of the fiber from the order of a micrometer down to the order of a nanometer. Shortening the diameter of the optical fiber provides access for the evanescent waves of the mode propagating through the tapered region. Given the evanescent wave intensity, $E$ decays exponentially from the distance, $a$, from the core-cladding interface, where $E_{0}$ is the initial evanescent wave and $b$ is the attenuation coefficient as expressed in (2):

$$
E=E_{0} \mathrm{e}^{-a b}
$$

Referring to (3), given $\lambda$ is the wavelength of the propagating light, $n_{2}$ and $n_{1}$ are the refractive indices of the glass and the medium surrounding it, and $\theta_{i}$ is the incident angle, thus the penetration depth $d_{p}$ is expressed as

$$
d_{p}=\frac{\lambda}{2 \pi \sqrt{n_{2}^{2} \sin ^{2} \theta_{i}-n_{1}^{2}}} .
$$

In (4), $d_{p}$ is defined as the depth at which the electric field drops to $1 / \mathrm{e}$ of the initial value at the surface. The number of reflections, $N$, happens at the glass-medium in the contact interface, depends greatly on the fiber diameter:

$$
N(\theta, d, l)=l * \frac{\tan (90-\theta)}{d}
$$

where $l$ is defined as the length between the fiber and the sample, and $\theta$ is the incident angle while $d$ is the fiber diameter in the sensing area. $N$ increases as $d$ decreases. From this context, it is concluded that the sensitivity of the fiber increases when the fiber diameter decreases.

\section{Sensor fabrication and experimental procedure}

\subsection{Fabrication of microfiber}

The tapered microstructure was fabricated by simultaneously heating and stretching a short section of a single-mode optical fiber (SMF). The process created a region of the fiber with a reduced and uniform diameter bounded by the conical unperturbed surrounding fiber line. The method is known as the heat and pull technique, and the setup is shown in Fig. 2(a). It is comprised of a linear guide stage, a moving stage, and a flame torch. The tapering system operates by pulling one side of the fiber while flame brushes it back and forth horizontally along the structure. Briefly, a standard single-mode silica optical fiber (model G652D) with core and cladding diameters of 10 microns and 125 microns was tapered. The polymer buffer coating was removed from a 2 -cm-long section in the middle of a 500-cm-long of the SMF using a mechanical stripper. The stripped section of the optical fiber was exposed to a flame produced by a butane gas burner ( $\max$ temperature $1800{ }^{\circ} \mathrm{C}$ ). A high precision stepper motor (model SD02B) and a microcontroller controlled the speed and moved the direction of the linear and moving stages. In this experiment, the pulling speed of the fiber holding blocks was kept at a constant rate of $1 \mathrm{~mm} / \mathrm{s}$ while the distance for the flame brushing was set at $1.5 \mathrm{~cm}$. The tapered optical fiber with a tapered length of 
$28 \mathrm{~mm}$ and a waist diameter of $9.03 \mu \mathrm{m}$ was fabricated, as shown in Fig. 2(b).

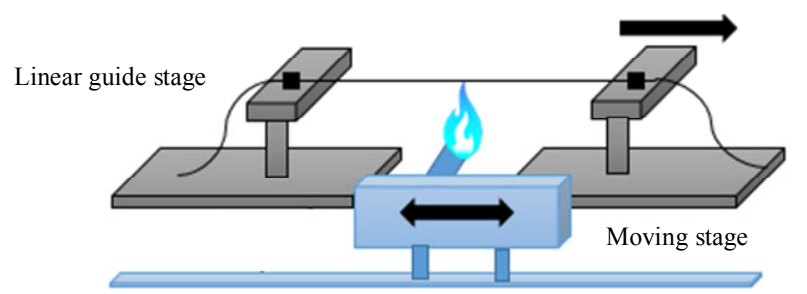

(a)

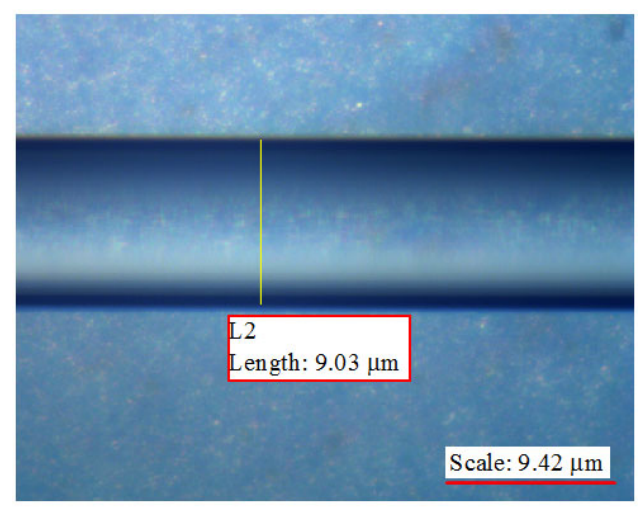

(b)

Fig. 2 Figures show (a) the tapering system established in the laboratory and (b) the structure of the tapered SMF observed under the microscope at 100/1.25 resolution with a diameter of $9.03 \mu \mathrm{m}$.

\subsection{Preparation of the GO-PVA composite and its deposition onto the fiber}

Commercially purchased GO dispersion (from Graphene Supermarket) with a concentration of $6 \mathrm{mg} / \mathrm{ml}$ and a lateral flake size in the range of $0.3 \mu \mathrm{m}-0.7 \mu \mathrm{m}$ was dissolved in deionized water until it reached the concentration of $2 \mathrm{mg} / \mathrm{ml}$. The solution was then mixed with the PVA in the powder form (Sigma Aldrich) with different ratios of $0.2 \mathrm{~g}$, $0.3 \mathrm{~g}$, and $0.4 \mathrm{~g}$. A homogenous mixture of the GO/PVA composite was obtained after it was stirred and heated for 12 hours in $30^{\circ} \mathrm{C}$. The tapered fiber was cleaned by dipping the tapered region in the acetone solution for 15 minutes. It was immersed into GO/PVA dispersion for 30 minutes before being left to dry for 24 hours at room temperature to allow the formation of the stable composite film on the surface of the fiber.

\subsection{Experimental setup}

The experimental setup for the proposed sensor is shown in Fig. 3. A Tungsten-Halogen light source (Ocean Optics HL-2000) with a wavelength range of $360 \mathrm{~nm}-2400 \mathrm{~nm}$ was utilized as the input light. The sensing region of the tapered fiber coated with the GO/PVA composite film was fixed in the sealed chamber. Thorlabs spectrometer CCS 200 series detected the output spectrum with the wavelength range of $200 \mathrm{~nm}-1000 \mathrm{~nm}$. The probe of a humidity meter with a sensitivity of $0.1 \mathrm{RH}(\%)$ was placed in the middle of the chamber to provide a reference. The humidity level in the chamber increased gradually with the presence of the saturated salt solution and decreased gradually when silica gel was added into the system. The GO/PVA composite film was moisture sensitive. Hence, changes in the RH inside the chamber would give a different result to the output spectrum. In this experiment, the performance of the sensor was investigated for RH ranging from $20 \%$ to $99.9 \%$. Any unnecessary movement was avoided during the data intake to preserve the setup adjustment.

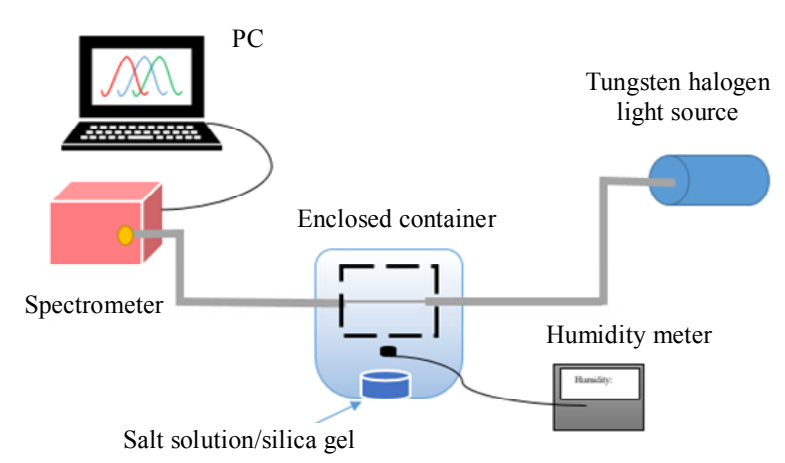

Fig. 3 Schematic diagram of the experimental setup.

\section{Results and discussion}

\subsection{FTIR analysis}

To further observe the hydrogen bonding interactions between the GO and PVA, Fourier transform infrared spectroscopy (FTIR) tests were conducted. Samples of GO, PVA, and GO/PVA nanocomposites in different compositions were 
characterized just before the coating process occurred. Figure 4 shows the characteristic transmission bands of the amorphous pure PVA, aqueous GO, and aqueous GO/PVA nanocomposite with different comprises of the PVA, $0.2 \mathrm{~g}, 0.3 \mathrm{~g}$, and $0.4 \mathrm{~g}$, respectively.

The FTIR spectrum of the PVA showed a broad absorption band with the peak located at $3351 \mathrm{~cm}^{-1}$ attributed to the symmetrical stretching vibration of $\mathrm{O}-\mathrm{H}$ while the peak at $1722 \mathrm{~cm}^{-1}$ corresponded to $\mathrm{C}=\mathrm{C}$ skeletal stretching vibration groups in the PVA [19]. The spectrum of the pure GO exhibited two main signature peaks located at $3323 \mathrm{~cm}^{-1}$ and $1635 \mathrm{~cm}^{-1}$ corresponding to hydroxyl $(\mathrm{O}-\mathrm{H})$ and carboxyl groups $(\mathrm{C}=\mathrm{O})$ stretches [20]. In the spectrum of well-dispersed PVA/GO nanocomposites, a broad and strong absorption band was observed between $3000 \mathrm{~cm}^{-1}$ and $3700 \mathrm{~cm}^{-1}$. As a general rule, the $\mathrm{O}-\mathrm{H}$ stretching peak was sensitive to the hydrogen bonding. The absorption band was slightly shifted from $3323 \mathrm{~cm}^{-1}$ in the pure GO to a lower wavenumber of $3315 \mathrm{~cm}^{-1}, 3299 \mathrm{~cm}^{-1}$, and $3290 \mathrm{~cm}^{-1}$ with the addition of $0.2 \mathrm{~g}, 0.3 \mathrm{~g}$, and $0.4 \mathrm{~g}$ of the PVA, indicating the formation of the hydrogen bonding between GO sheets and additional PVA chains.

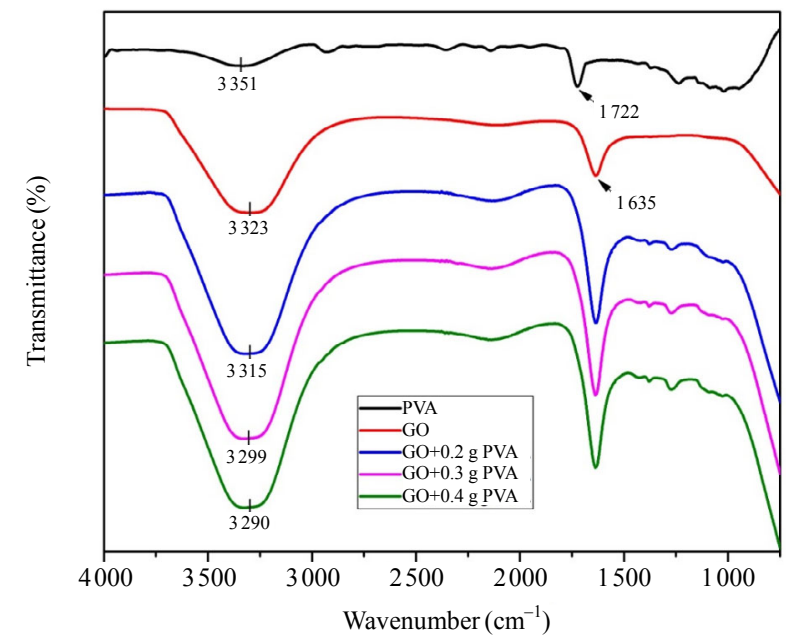

Fig. 4 FTIR curves of the PVA, GO, $10 \mathrm{ml} \mathrm{GO}+0.2 \mathrm{~g}$ PVA, $10 \mathrm{ml} \mathrm{GO}+0.3 \mathrm{~g} \mathrm{PVA}$, and $10 \mathrm{ml} \mathrm{GO}+0.4 \mathrm{~g}$ PVA.
Meanwhile, the peak for carboxyl stretching vibration at $1635 \mathrm{~cm}^{-1}$ appeared with a higher intensity in the GO/PVA spectrum, indicating that the hydrogen bonding between $\mathrm{C}=\mathrm{O}$ and $\mathrm{O}-\mathrm{H}$ has been established. The results suggested that the addition of the PVA damaged the GO structure by introducing hydrogen bonding interactions between the hydroxyl groups on the PVA molecular chains and the oxygen-containing functional groups of GO sheets as referred to Fig. 1. Strong interaction between the GO and PVA confirmed the good interfacial adhesion, which is an important criterion for the nanocomposite to be used as the coatings layer of the sensor.

\subsection{Surface morphology}

The surface morphology of the GO/PVA nanocomposite coated on tapered optical fibers was inspected by the field emission scanning electron microscopy (FESEM, ZEISS SIGMA). Figure 5(a) shows an overview of the surfaces of the GO/PVA composite as the top layer coated on the fiber. The nanocomposites surface appears to be uniformly distributed under the low magnification image, indicating that the PVA was homogeneously dispersed into the GO layers. Figure 5(b) shows the surface of the coated tapered fiber at $100 \mathrm{~K}$ magnification. In contrast, the surfaces of the nanocomposites showed noticeable roughness with minimal cracks and no specific shape identified. The uniform coating had wrinkles appeared on it, and a common phenomenon was observed in 2D films and membranes. The grain structure was improved gradually with the increasing amount of the cross-linking network between the GO and PVA, resulting in the formation of the well-textured and oriented molecular structure of the surface. The coating deposited appeared to be uniform throughout the fiber with coating thickness of $82.94 \mathrm{~nm}$ to $84.56 \mathrm{~nm}$ as illustrated in Fig. 5(c). 


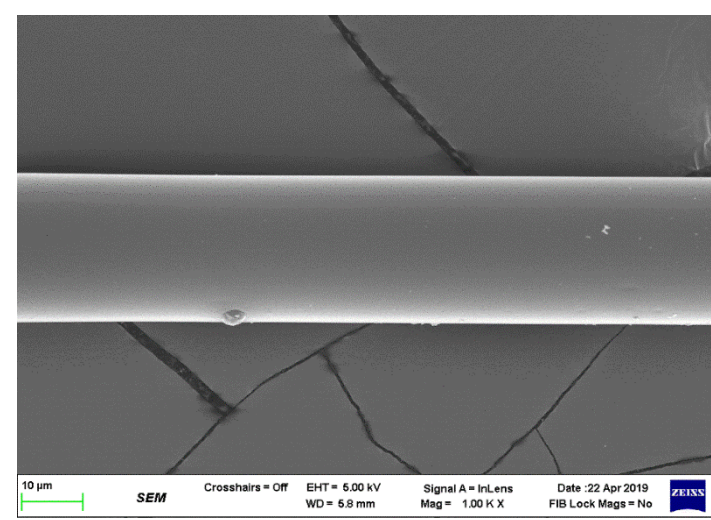

(a)

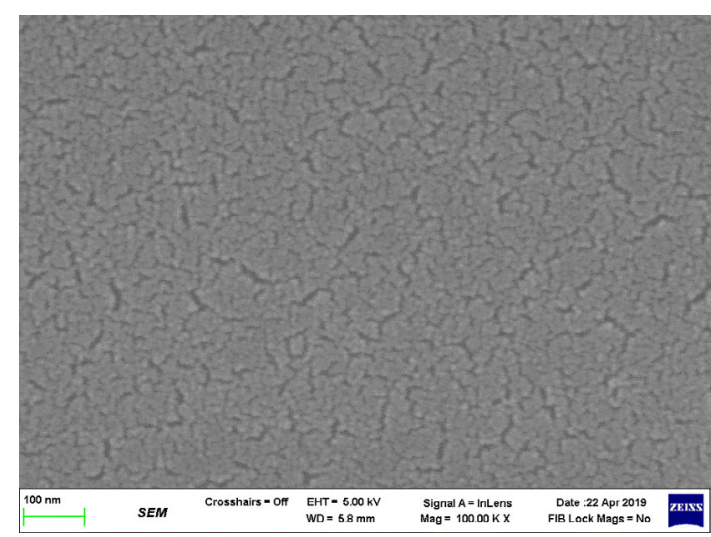

(b)

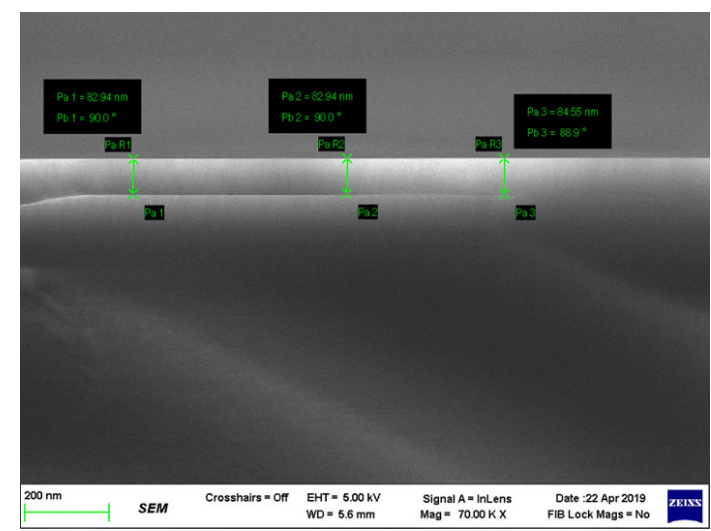

(c)

Fig. 5 FESEM: (a) top view image of the tapered fiber coated with the GO/PVA nanocomposite layer, (b) magnified top view image of the tapered fiber coated with the GO/PVA nanocomposite layer, and (c) cross-sectional view of the tapered fiber coated with the GO/PVA nanocomposite layer.

\subsection{Raman analysis}

The GO/PVA nanocomposite film on the tapered fiber had undergone characterization through the Raman spectroscopy to identify the molecules present on the nanocomposite coating on the tapered fiber. Figure 6 shows the Raman spectrum of the
GO/PVA nanocomposite measured at room temperature with a $532 \mathrm{~nm}$ laser excitation.

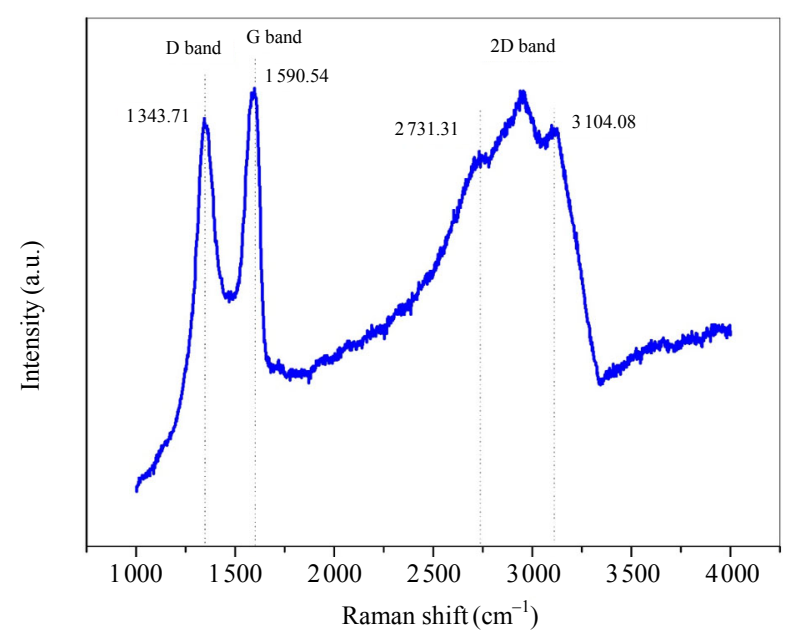

Fig. 6 Raman spectrum of the coated tapered fiber.

Three significant peaks could be observed from the characteristic curve. The D peak was located at $1343.71 \mathrm{~cm}^{-1}$ due to stretching of the $\mathrm{C}-\mathrm{C}$ bonding from the doubly resonant-induced mode [21]. The $\mathrm{G}$ peak appeared at the Brillouin zone center 1 $595.76 \mathrm{~cm}^{-1}$ where it was due to doubly degenerate phonon mode because of the first-order scattering of $\mathrm{E}_{2 \mathrm{~g}}$ of phonon of $\mathrm{sp}^{2} \mathrm{C}$ atoms [22]. The two prominent defects induced that the selected GO had a large defect density. The 2D band was located at $2716.56 \mathrm{~cm}^{-1}$. The value of relative intensity ratio of both the $\mathrm{D}$ and $\mathrm{G}$ peaks $\left(I_{D} / I_{G}\right)$ defined by the measure of disorder degree [23] was 0.93 . Intensity of the $2 \mathrm{D}$ peak to the intensity of the G peak $\left(I_{2 \mathrm{D}} / I_{G}\right)$ gave information about the number of graphene layers in the material [24], which in this case was 0.89 . These values indicated few-layer graphene oxide presence in the GO/PVA composite coating.

\subsection{Humidity sensing mechanism}

The sensing mechanism of this device was based on the adoption of the GO/PVA thin film on the optical fiber tapered region. Dispersion of the layered GO into PVA matrix formed stable nanocomposites through the strong hydrogen bonding. Due to the polyelectrolyte nature of the PVA, the nanocomposites could be exploited as a 
moisture-sensitive functional layer. The presence of $\mathrm{RH}$ from ambient surrounding resulted in water molecule accommodation within the composites interlayer. The coating layer swelled and its inertial mass increased, thus, altering the effective refractive index of the coating material. The variation in effective refractive index of the coating would subsequently alter the light transmission as suggested by the total internal reflection principle. Figure 7 illustrates the absorption spectra of tapered optical fibers coated with (a) $10 \mathrm{ml} \mathrm{GO}+0.2 \mathrm{~g}$ PVA, (b) $10 \mathrm{ml} \mathrm{GO}+0.3 \mathrm{~g}$ PVA, and (c) $10 \mathrm{ml} \mathrm{GO}+0.4 \mathrm{~g}$ PVA composite films under different surrounding RHs ranging from $20 \%$ to $99.9 \%$.

Figure 7(a) shows that the spectral response from $10 \mathrm{ml} \mathrm{GO}+0.2 \mathrm{~g}$ PVA coating was inconsistent and not in particular increasing or decreasing manners concerning surrounding RH. Total absorption percentage was recorded in a small range between $85 \%$ and $93 \%$. There were two obvious overlapping spectra observed between $20 \% \mathrm{RH}$ and $30 \% \mathrm{RH}$, and $50 \% \mathrm{RH}$ and $60 \% \mathrm{RH}$, respectively. The unstable signals from the sensor were mainly due to the non-uniform composite structure formed by relatively low amount of the PVA involved. A small fraction of PVA molecules were intercalated into the three-dimension interconnected GO structure, and the remaining PVA inevitably passed through the layers resulting in the instability of the composite structure.

A linear relationship between the absorbance and surrounding RH is shown in Fig. 7(b) recorded from the sensor coated with $10 \mathrm{ml}$ GO $+0.3 \mathrm{~g}$ PVA composite. As the humidity increased, the percentage of absorption decreased in the wide range of $5 \%$ to $60 \%$. In this case, a significant absorption capability of the composite layers was shown. A satisfactory amount of PVA molecules were supplied and intercalated with the GO structure, and acted as ideal bridges in the GO/PVA composite. As a result, more hydrophilic surfaces were created and favored the water molecule absorption ability of the coating upon exposure towards humid surrounding. The absorbed water molecules filled the slices spaces between the PVA and GO layers, leading to the swell of the GO/PVA composite structure. This interaction was responsible for a decrease in the effective refractive index of coating layers and further alteration of transmitted light intensity.

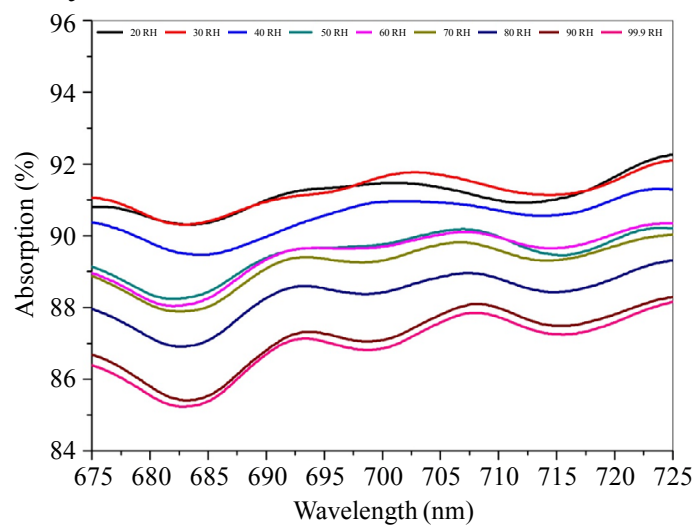

(a)

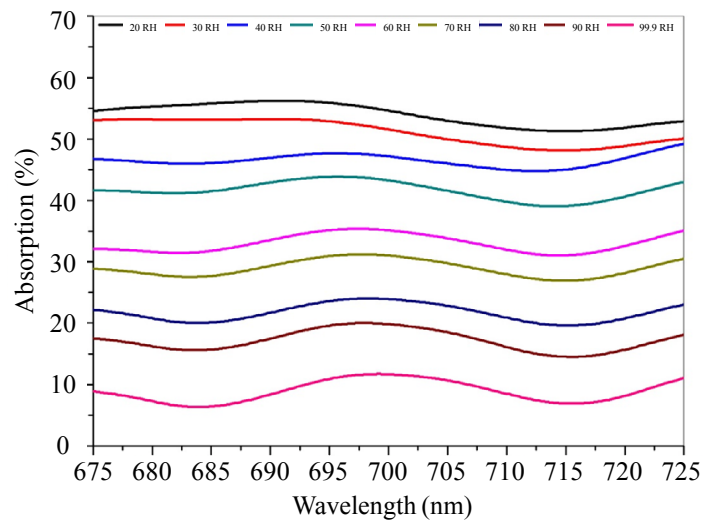

(b)

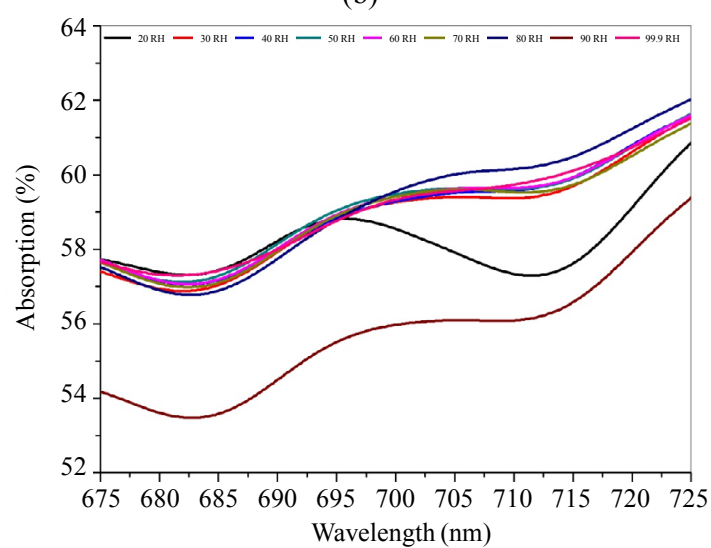

(c)

Fig. 7 Percent absorptions of the tapered optical fiber coated with (a) $10 \mathrm{ml} \mathrm{GO}+0.2 \mathrm{~g}$ PVA, (b) $10 \mathrm{ml} \mathrm{GO}+0.3 \mathrm{~g}$ PVA, and (c) $10 \mathrm{ml} \mathrm{GO}+0.4 \mathrm{~g}$ PVA composite films under RH ranging from $20 \%$ to $99.9 \%$. 
Figure 7(c) shows an inconsistent spectral response again being monitored when the sensor was coated with $10 \mathrm{ml} \mathrm{GO}+0.4 \mathrm{~g}$ PVA. The total absorption percentage was recorded in relatively small range between $53 \%$ and $62 \%$. An increase in the PVA resulted in the gallery spacing presented within the GO layer, which was gradually filled and blocked by entangled PVA polymer chains. The defect introduced within the composite layers would impede the ability of the GO/PVA coating to absorb water molecules, thus resulting in a poor efficiency of the sensor. The correlation between the percent relative absorption and percent $\mathrm{RH}$ is plotted in Fig. 8 with the fitting line. The highest sensitivity recorded was $0.5290 \mathrm{RH}(\%)$ corresponding to the composite film of $10 \mathrm{ml} \mathrm{GO}+0.3 \mathrm{~g}$ PVA whereas the composite films with the values of the PVA $0.2 \mathrm{~g}$ and $0.4 \mathrm{~g}$ had the low sensitivities of $0.0659 \mathrm{RH}(\%)$ and $0.0077 \mathrm{RH}(\%)$, respectively.

A series of experiments were carried out in terms of repeatability, reversibility, stability, and response time of the chosen sensor with the composite film of $10 \mathrm{ml} \mathrm{GO}+0.3 \mathrm{~g}$ PVA to further investigate its performance. Repetitive experiments of the proposed humidity sensor were conducted to observe the accuracy of the data executed by the sensor. The experiment results are shown in Fig. 9 where the points are quite close to each other showing high accuracy data with the maximum sensitivity error of $3.11 \%$ in three trials. The fabricated sensor was tested for its reversibility, and the result is shown in Fig. 10. The data of the same humidity in increasing and decreasing orders fell nearly at the same point, indicating a good reversibility. The reversibility was monitored when the $\mathrm{RH}$ level changed from $20 \% \mathrm{RH}$ to $99.9 \% \mathrm{RH}$; both of these RH levels were controlled using saturated salt solution and silica gel. The enclosed container was designed to allow the humidity level in the container to switch from one to another.

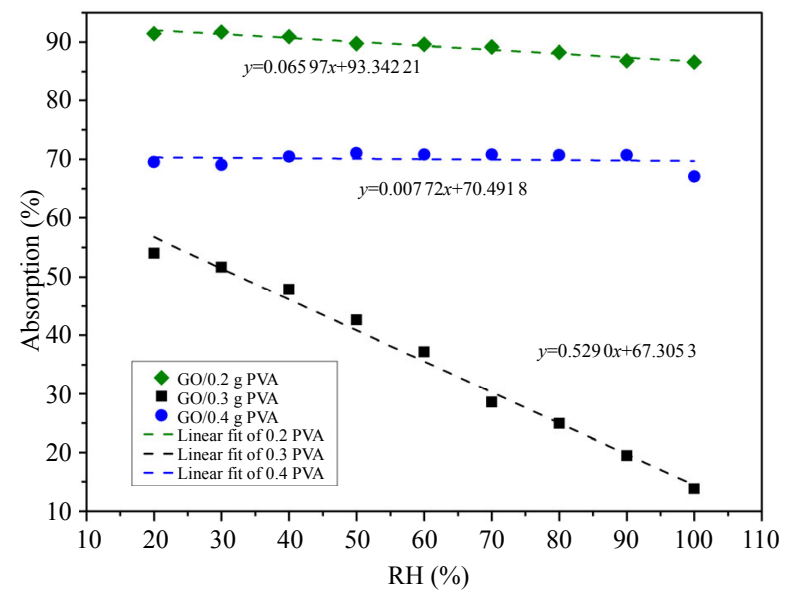

Fig. 8 Fitting line of the sensitivity of the sensor coating with different compositions at the wavelength of $700 \mathrm{~nm}$.

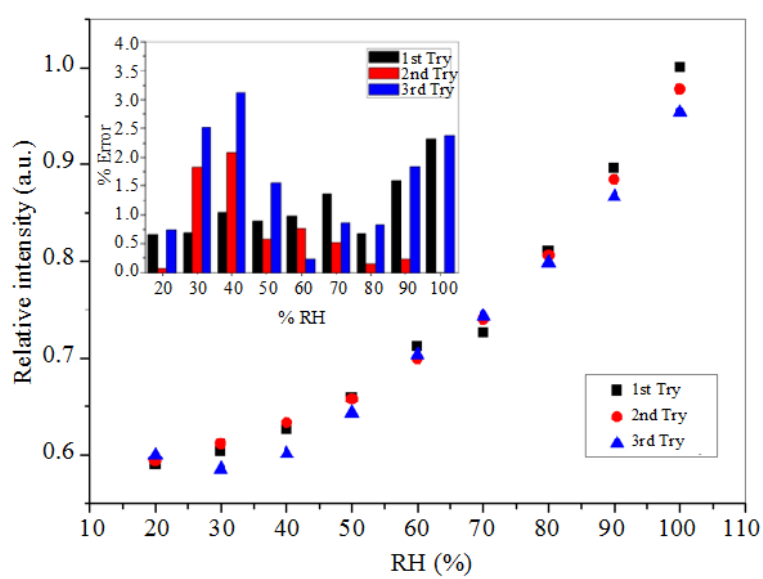

Fig. 9 Repetitive test result of the tapered fiber sensor coated with the GO/PVA composite.

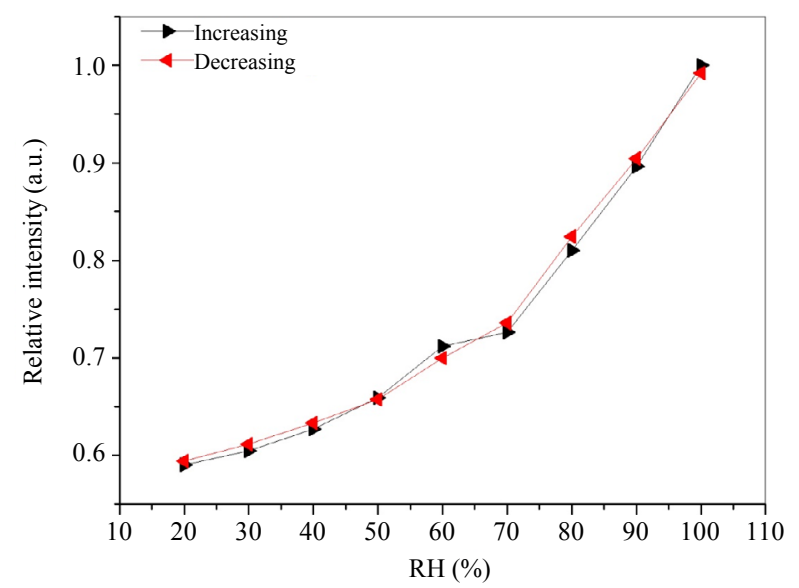

Fig. 10 Reversibility of the sensor at room temperature.

The stability of the proposed humidity sensor coated with the GO/PVA composite film was tested for 90 minutes under three fixed RH levels of $20 \%$ 
RH (the lowest \% RH), 65\% RH (room \% RH), and 99.9\% RH (the highest \% RH), respectively. Based on the results shown in Fig. 11, there were only small fluctuations that occurred in the three conditions. Light source fluctuation might be one of the factors affecting the stability. However, this could be much negligible over a large humidity change. Fluctuation happened in the range of 0.004 for $20 \% \mathrm{RH}$ as shown in Fig. 12(a) and 0.005 when the sensor operated at room \% RH $(65 \% \mathrm{RH})$, as shown in Fig. 12(b). The highest fluctuation happened at $99.9 \% \mathrm{RH}$ where the data fluctuated in the range of 0.009 , as shown in Fig. 12(c). A self-referencing based on the single micro bend loop technique as per described in [25] could be used instead of using the halogen-tungsten lamp to avoid any fluctuations effecting the data. The second optical source was unaffected by the measurement technique, hence the output is undependable of the source intensities.

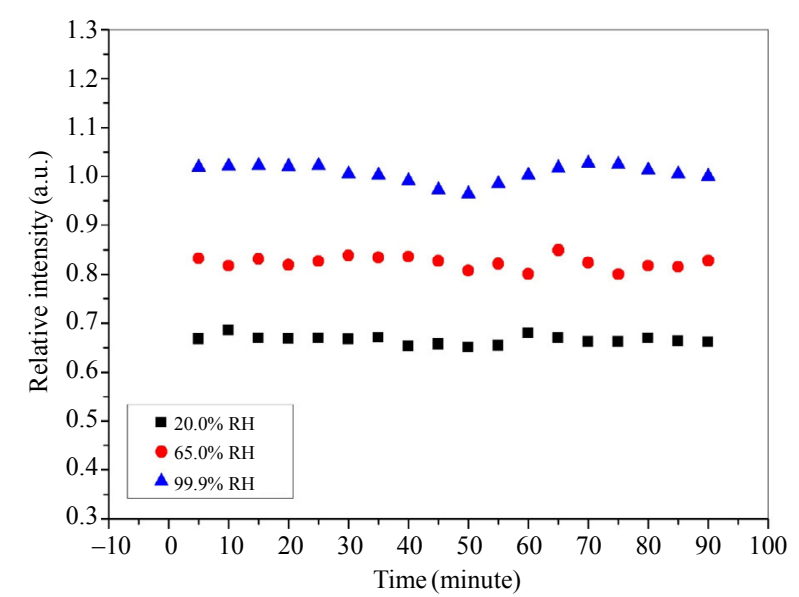

Fig. 11 Stability test results of the proposed sensor at three different RHs.

A double $y$-axis graph is plotted in Fig. 13 to study the effect of power in response to increasing value of relative humidity. The response time of the system was evaluated by connecting the coated tapered fiber with a power meter to measure the power $(\mathrm{nW})$ output of the sensor in correlation with time and RH. The response time was monitored in a controlled environment of $25^{\circ} \mathrm{C}$. The time taken for the sensor to reach $99.9 \% \mathrm{RH}$ level was 147 seconds. The recovery time was also investigated as plotted in Fig. 14, in which the value was 293 seconds.

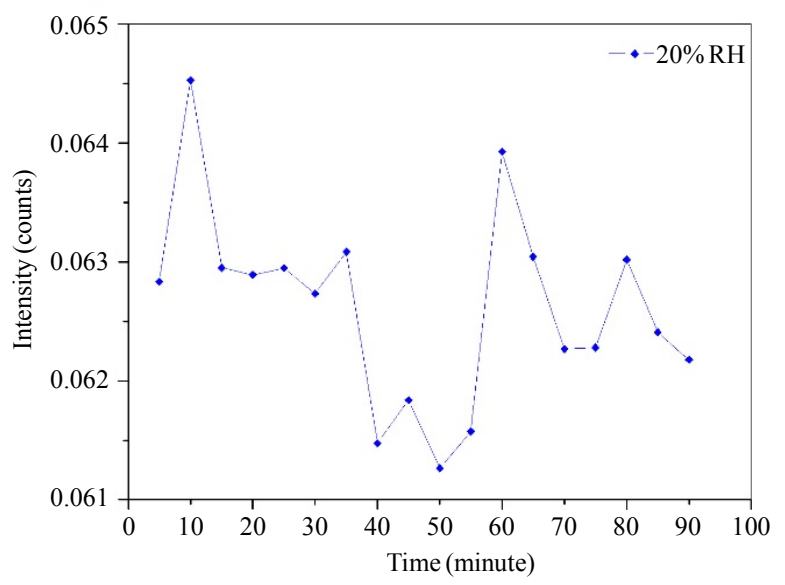

(a)

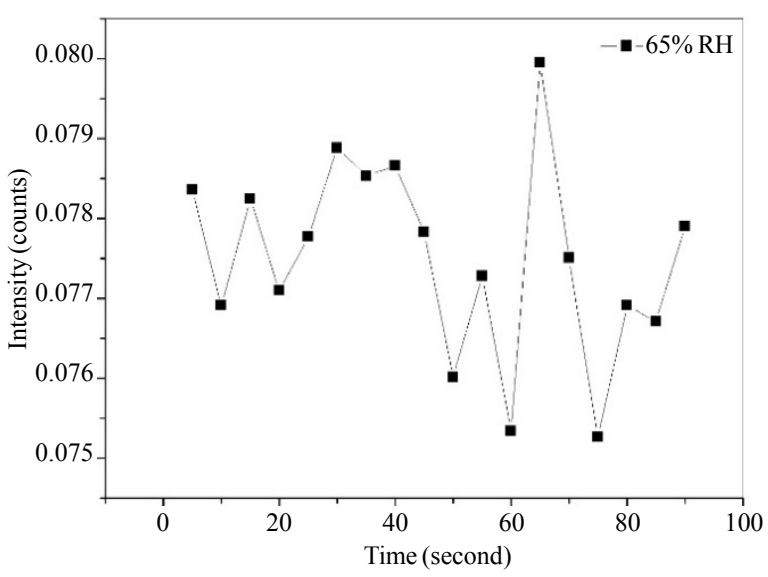

(b)

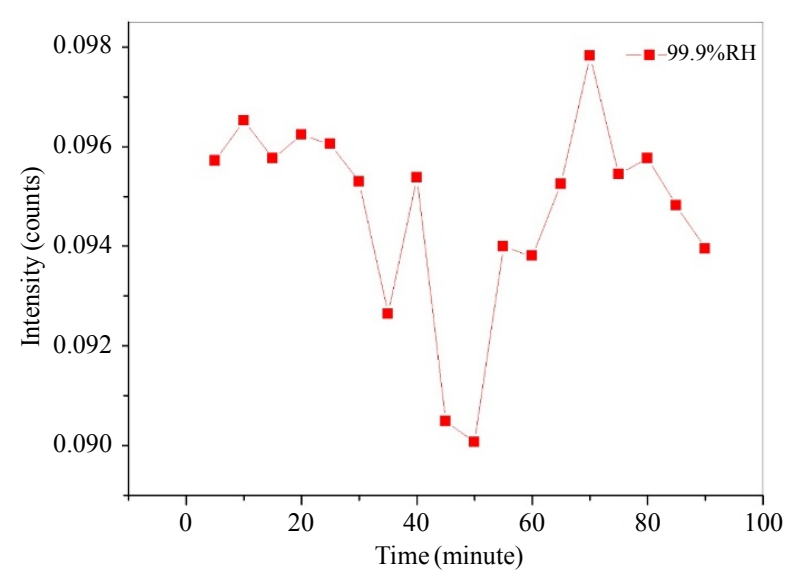

(c)

Fig. 12 Fluctuation of stability data at (a) $20 \% \mathrm{RH}$, (b) $65 \% \mathrm{RH}$, and (c) $99.9 \% \mathrm{RH}$. 


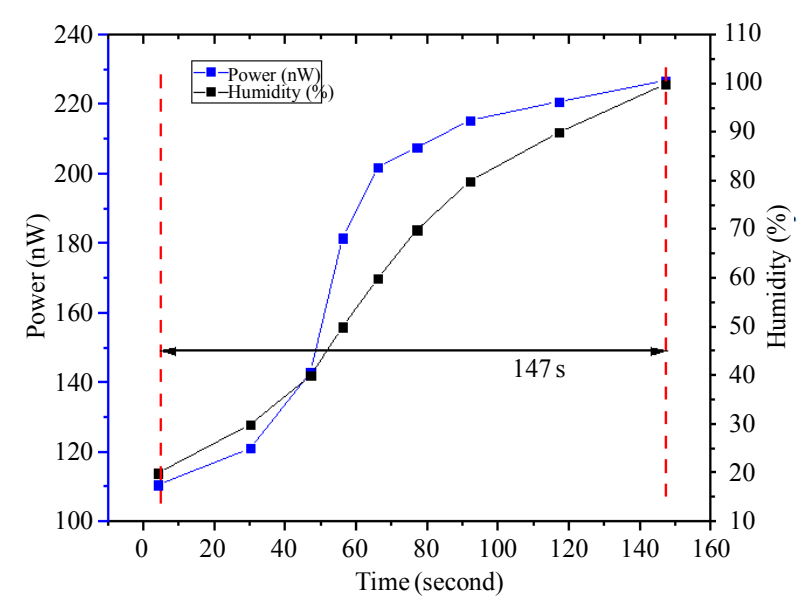

Fig. 13 Response time of the designated sensor.

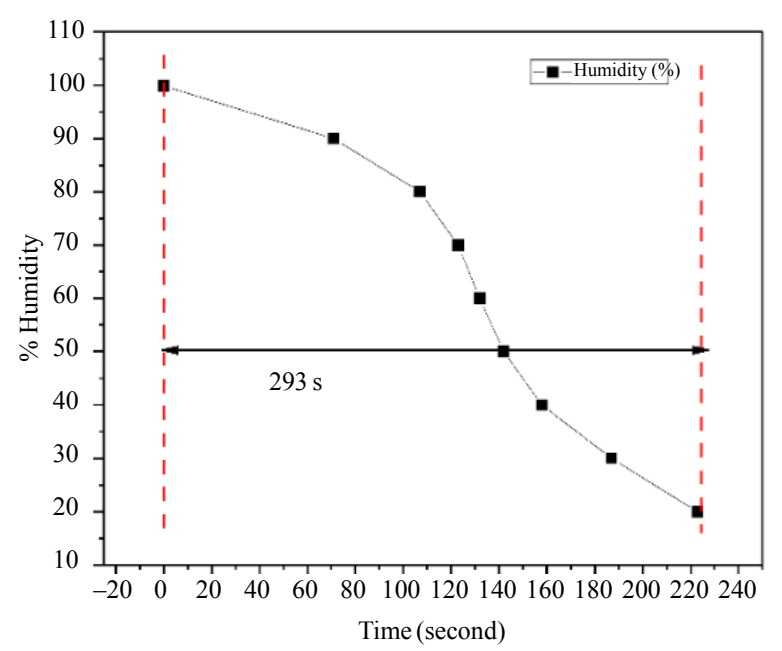

Fig. 14 Recovery time of the sensor.

\section{Conclusions}

The response of the tapered fiber coated with the GO/PVA composite film for RH sensing purpose was investigated. The PVA was chosen as the coating material due to its excellent property of good swelling rate with moisture. GO layers on the other hand possessed a high surface area for the intercalation of the PVA, thus making the mixture of both complements each other as a good composite film to be coated on the fiber sensor. Three sensors with different compositions were tested under $\mathrm{RH}$ ranging from $20 \% \mathrm{RH}$ to $99.9 \% \mathrm{RH}$ to figure out the right ratio of the GO and PVA used as the coating film. For comparison, the fiber sensor with a coating of $10 \mathrm{ml} \mathrm{GO}+0.3 \mathrm{~g}$ PVA showed the highest sensitivity of $0.5290 \mathrm{RH}(\%)$. Repeatability, reversibility, stability, and response time of the designated sensor were in good measurement, attributing to a reliable sensor.

\section{Acknowledgment}

The authors would like to thank Laser Centre, Ibnu-Sina Institute for Scientific and Industrial Research (ISI-SIR), Universiti Teknologi Malaysia (UTM) for the support. This research work has been supported by UTM_TDR 06G13 research grant. Aneez SYUHADA and Muhammad Salleh SHAMSUDIN acknowledge the funding from the UTM Zamalah Scholarship Award supported by the School of Graduate Studies, UTM. The authors want to thank the critics of the anonymous reviewers and valuable suggestions for improving this manuscript.

Open Access This article is distributed under the terms of the Creative Commons Attribution 4.0 International License (http://creativecommons.org/licenses/by/4.0/), which permits unrestricted use, distribution, and reproduction in any medium, provided you give appropriate credit to the original author(s) and the source, provide a link to the Creative Commons license, and indicate if changes were made.

\section{References}

[1] H. Farahani, R. Wagiran, and M. N. Hamidon, "Humidity sensors principle, mechanism, and fabrication technologies: a comprehensive review," Sensors, 2014, 14(5): 7881-7939.

[2] T. Liu, Y. Wei, G. Song, Y. Li, J. Wang, Y. Ning, et al., "Advances of optical fiber sensors for coal mine safety monitoring applications," in International Conference on Microwave and Photonics (ICMAP), India, Dec. 13-15, 2013, pp. $1-5$.

[3] D. R. Raj, S. Prasanth, T. V. Vineeshkumar, and C. Sudarsanakumar, "Ammonia sensing properties of tapered plastic optical fiber coated with silver nanoparticles/PVP/PVA hybrid," Optics Communications, 2015, 340: 86-92.

[4] K. K. K. Annamdas and V. G. M. Annamdas, "Review on developments in fiber optical sensors and applications," in SPIE Defense, Security, and Sensing, USA, April 23, 2010, pp. 76770R-176770R-12.

[5] J. Ascorbe, J. M. Corres, F. J. Arregui, and I. R. Matias, "Recent developments in fiber optics 
humidity sensors," Sensors, 2017, 17(4): 893-915.

[6] S. Novais, M. S. Ferreira, and J. L. Pinto, "Relative humidity fiber sensor based on multimode interferometer coated with agarose-gel," Coatings, 2018, 8(12): 453-461.

[7] C. Zhao, Q. Yuan, L. Fang, X. Gan, and J. Zhao, "High-performance humidity sensor based on a polyvinyl alcohol-coated photonic crystal cavity," Optics Letters, 2016, 41(23): 5515-5518.

[8] B. Deshkulkarni, L. R. Viannie, S. V. Ganachari, N. R. Banapurmath, and A. Shettar, "Humidity sensing using polyaniline/polyvinyl alcohol nanocomposite blend," in International Conference on Advances in Manufacturing, Materials and Energy Engineering (ICon MMEE 2018), India, March 2-3, 2018, pp. $1-8$.

[9] P. Wang, K. Ni, B. Wang, Q. Ma, and W. Tian, “A chitosan-coated humidity sensor based on Michelson interferometer with thin-core optical fiber," in 2017 16th International Conference on Optical Communications and Networks (ICOCN), China, Aug. 7-10, 2017, pp. 1-3.

[10] J. Ascorbe, J. M. Corres, F. J. Arregui, and I. R. Matias, "Optical fiber humidity sensor based on a tapered fiber asymmetrically coated with indium tin oxide," in Sensors, 2014 IEEE, Spain, Nov. 2-5, 2014, pp. 1916-1919.

[11] X. Li, X. Chen, X. Chen, X. Ding, and X. Zhao, "High-sensitive humidity sensor based on graphene oxide with evenly dispersed multiwalled carbon nanotubes," Materials Chemistry and Physics, 2018, 207: 135-140.

[12] M. Shojaee, Sh. Nasresfahani, M. K. Dordane, and M. H. Sheikhi, "Fully integrated wearable humidity sensor based on hydrothermally synthesized partially reduced graphene oxide," Sensors and Actuators A: Physical, 2018, 279: 448-456.

[13] M. Donarelli and L. Ottaviano, "2D materials for gas sensing applications: a review on graphene oxide, $\mathrm{MoS}_{2}, \mathrm{WS}_{2}$ and phosphorene," Sensors, 2018, 18(11): 3638-3682.

[14] C. Sun, Q. Shi, M. S. Yazici, C. Lee, and Y. Liu, "Development of a highly sensitive humidity sensor based on a piezoelectric micromachined ultrasonic transducer array functionalized with graphene oxide thin film," Sensors, 2018, 18(12): 43524363.

[15] U. Stahl, A. Voigt, M. Dirschka, N. Barié, C. Richter, A. Waldbaur, et al., "Long-term stability of polymer-coated surface transverse wave sensors for the detection of organic solvent vapors," Sensors, 2017, 17(11): 2529-2541.

[16]M. Cano, U. Khan, T. Sainsbury, A. O’Neil, Z. Wang, I. T. McGovern, et al., "Improving the mechanical properties of graphene oxide based materials by covalent attachment of polymer chains," Carbon, 2013, 52: 363-371.

[17] L. Zhang, Z. Wang, C. Xu, Y. Li, J. Gao, W. Wang, et al., "High strength graphene oxide/polyvinyl alcohol composite hydrogels," Journal of Materials Chemistry, 2011, 21(28): 10399-10406.

[18] X. Yang, S. Shang, and L. Li, "Layer - structured poly (vinyl alcohol)/graphene oxide nanocomposites with improved thermal and mechanical properties," Journal of Applied Polymer Science, 2011, 120(3): 1355-1360.

[19]A. A. R. Oliveiraa, V. S. Gomidea, M. F. Leiteb, H. S. Mansura, and M. M. Pereira, "Effect of polyvinyl alcohol content and after synthesis neutralization on structure, mechanical properties and cytotoxicity of sol-gel derived hybrid foams," Materials Research, 2009, 12(2): 239-244.

[20] T. Rattana, S. Chaiyakun, N. Watit-anun, N. Nuntawong, P. Chindaudom, S. Oaew, et al., "Preparation and characterization of graphene oxide nanosheets," Procedia Engineering, 2012, 32: 759-764.

[21] C. Thomsen and S. Reich, "Double resonant Raman scattering in graphite," Physical Review Letters, 2000, 85(24): 5214-5217.

[22] A. C. Ferrari and J. Robertson, "Interpretation of Raman spectra of disordered and amorphous carbon," Physical Review B, 2000, 61(20): 14095-14107.

[23] G. Sobon, J. Sotor, J. Jagiello, R. Kozinski, M. Zdrojek, M. Holdynski, et al., "Graphene oxide vs. reduced graphene oxide as saturable absorbers for Er-doped passively mode-locked fiber laser," Optics Express, 2012, 20(17): 19463-19473.

[24] R. Muzyka, S. Drewniak, T. Pustelny, M. Chrubasik, and G. Gryglewicz, "Characterization of graphite oxide and reduced graphene oxide obtained from different graphite precursors and oxidized by different methods using Raman spectroscopy," Materials, 2018, 11(7): 1050-1064.

[25] A. Tapetado, P. J. Pinzón, J. Zubia, and C. Vázquez, "Polymer optical fiber temperature sensor with dual-wavelength compensation of power fluctuations," Journal of Lightwave Technology, 2015, 33(13): 2716-2723. 